\title{
A POLYNOMIAL-TIME TEST FOR TOTAL DUAL INTEGRALITY IN FIXED DIMENSION
}

\author{
W. COOK
}

Department of Combinatorics and Optimization, University of Waterloo, Waterloo, Ontario, Canada N2L $3 G 1$

\section{LOVÁSZ,}

Analizis I. tszk., Eötvös Lorand Tudományegyetem, Muzeum krt. 6-8, H-1088, Budapest, Hungary

\section{A. SCHRIJVER,}

Department of Econometrics, Tilburg University, P.O. Box 90153, Tilburg, Holland

Received 2 December 1982

Revised manuscript received 27 June 1983

\begin{abstract}
In this note we show that, for any fixed number $r$, there exists a polynomial-time algorithm to test whether a given system of linear inequalities $A x \leqslant b$ is totally dual integral, where $A$ is an integer matrix of rank $r$.
\end{abstract}

Key words: Polynomial-Time, Algorithm, Total Dual Integrality, Hilbert Basis, Integer Linear Programming.

\section{Introduction}

Let $A$ be a rational $m \times n$-matrix, and let $b$ be a rational vector of length $m$. The system $A x \leqslant b$ of linear inequalities is called totally dual integral if the minimum in the linear programming duality equation

$$
\max \{c x \mid A x \leqslant b\}=\min \{y b \mid y \geqslant 0, y A=c\}
$$

has an integer optimal solution for each integer vector $c$ for which the optima exist ( $c x$ and $y b$ denoting inner products). Edmonds and Giles [3] motivated this concept by showing that if $A x \leqslant b$ is totally dual integral and $b$ is integer, then also the maximum in (1) has an integer optimal solution, for each vector $c$ for which the optima exist. Equivalently, then the polyhedron

$$
P=\{x \mid A x \leqslant b\}
$$

is the convex hull of the lattice points contained in it.

In this note we show that, for any fixed number $r$, there exists an algorithm which tests the total dual integrality of a given system $A x \leqslant b$ of linear inequalities, where $A$ is an integer matrix of rank $r$, in time bounded by a polynomial in the sizes of 
$A$ and $b$ (in binary notation). The main tool here is Lenstra's polynomial-time algorithm for integer linear programming in fixed dimension [7].

Total dual integrality is closely connected to so-called 'Hilbert bases'. Following Giles and Pulleyblank [4], a set of integer vectors $a_{1}, \ldots, a_{k}$ is called a Hilbert basis if each integer vector in the convex cone spanned by $a_{1}, \ldots, a_{k}$ is a nonnegative integer combination of $a_{1}, \ldots, a_{k}$. Indeed, the system $A x \leqslant b$ is totally dual integral if and only if for each minimal face $F$ of $P$ the rows of $A$ which are active in $F$ form a Hilbert basis (a row is active in $F$ if the corresponding inequality in $A x \leqslant b$ is satisfied with equality by each vector in $F$ ). In particular, all rows of $A$ form a Hilbert basis if and only if the system $A x \leqslant 0$ is totally dual integral.

It is easy to see that each pointed rational polyhedral cone $C$ is spanned by a unique minimal Hilbert basis (minimal under inclusion)-take all nonzero integer vectors in $C$ which cannot be expressed as a sum of other integer vectors in $C$ (this is a finite set, as these vectors are all contained in the polytope $\left\{\lambda_{1} b_{1}+\cdots+\lambda_{t} b_{t} \mid 0 \leqslant\right.$ $\left.\lambda_{i} \leqslant 1(1 \leqslant i \leqslant t)\right\}$, where $b_{1}, \ldots, b_{t}$ are integer vectors spanning $\left.C\right)$. This implies that each full-dimensional rational polyhedron is defined by a unique minimal totally dual integral system with integer left-hand sides (cf. [8]).

Since, if we fix the rank $r$ of $A$, the number of minimal faces of $P$ is polynomially bounded by the number of rows of $A$, and since they can be enumerated in polynomial time, testing total dual integrality for fixed rank in polynomial time can be reduced to the test of being a Hilbert basis for fixed rank in polynomial time.

Chandrasekharan [1] proved that there exists an algorithm which finds for any totally dual integral system $A x \leqslant b$, with $A$ integer, and for any integer vector $c$, an integer optimum solution $y$ for the minimum in (1), in time polynomially bounded by the sizes of $A, b$ and $c$ (in binary notation).

The property of being a totally dual integral system, and that of being a Hilbert basis, is in the class co-NP, if the left-hand side coefficients (i.e. the entries of $A$ ) are integral. That is, there exists a polynomial-length proof for not being totally dual integral, or for not being a Hilbert basis. Indeed, if the integer vectors $a_{1}, \ldots, a_{k}$ do not form a Hilbert basis, there exists an integer vector $c$ in the cone $C$ spanned by $a_{1}, \ldots, a_{k}$ such that $c-a_{i} \notin C$ for all $i$ for which $a_{i}$ does not belong to the minimal face $F$ of $C$, and such that $c$ does not belong to $F$. Note that, given a vector $c$, these conditions can be checked with Khachiyan's method. Moreover, $c$ can be chosen in the polytope $\left\{\lambda_{1} a_{1}+\cdots+\lambda_{k} a_{k} \mid 0 \leqslant \lambda_{i} \leqslant 1(1 \leqslant i \leqslant k)\right\}$, so that the size of $c$ is polynomially bounded by the size of $a_{1}, \ldots, a_{k}$.

In fact it is the content of the theorem that if we fix the rank of $a_{1}, \ldots, a_{k}$, such a vector $c$ can be found in polynomial time. (Parts of the proof of this theorem also occur in [2].)

Theorem. For any fixed natural number $r$, there exists an algorithm which tests, for any integer matrix $A$ of rank $r$ and for any rational vector $b$, whether the system $A x \leqslant b$ is totally dual integral, in time bounded by a polynomial in the sizes of $A$ and $b$ (in binary notation). 
Proof. I. Let $A$ have rows $a_{1}, \ldots, a_{m}$. We first argue that we may assume that $b=0$, and that the convex cone $C$ spanned by $a_{1}, \ldots, a_{m}$ is pointed and full-dimensional (equivalently, that the cone $\{x \mid A x \leqslant 0\}$ is pointed and full-dimensional).

Indeed, we have to test whether in each minimal face $F$ of $P=\{x \mid A x \leqslant b\}$, the active rows of $A$ form a Hilbert basis. Now we can enumerate all minimal faces of $P$ as follows. Enumerate all collections of $r$ linearly independent rows of $A$. If, say, $a_{1}, \ldots, a_{r}$ are linearly independent, we check whether $F=\left\{x \mid a_{1} x=b_{1}, \ldots, a_{r} x=b_{r}\right\}$ is contained in $P$, which can be done by checking whether $A x_{0} \leqslant b$ holds for some solution $x_{0}$ of $a_{1} x=b_{1}, \ldots, a_{r} x=b_{r}$ If $F \subseteq P$, then $F$ is a minimal face of $P$. Let, say, $a_{1} x \leqslant b_{1}, \ldots, a_{k} x \leqslant b_{k}$ be those inequalities among $A x \leqslant b$ which are active in $F$ (i.e., which hold with equality for some vector, and hence for each vector, in $F$ ). We have to test whether $a_{1}, \ldots, a_{k}$ is a Hilbert basis. That is, if $\boldsymbol{A}^{\prime}$ denotes the matrix with rows $a_{1}, \ldots, a_{k}$, we have to test whether $A^{\prime} x \leqslant 0$ is totally dual integral. Therefore, since for fixed $r$ the number of minimal faces is polynomially bounded by the size of $A$, we may assume that $b=0$.

Next we show that we may assume that the convex cone $C$ spanned by $a_{1}, \ldots, a_{m}$ is pointed and full-dimensional. Let $F$ be the unique minimal face of $C$, and let $L$ be the linear hull of $C$. Let, say, $a_{1}, \ldots, a_{t}$ be the rows of $A$ belonging to $F$ (they can be determined in polynomial time as a row $a_{i}$ of $A$ belongs to $F$ if and only if $-a_{i}$ belongs to $C$ ). Let $d:=\operatorname{dim} F$ (and note that $r=\operatorname{dim} L$ ). Now there exists a unimodular matrix $U$ such that $F U=\mathbb{R}^{d} \times 0^{n-d}$, and $L U=\mathbb{R}^{r} \times 0^{n-r}$. Such a matrix $U$ can be found as follows. Choose $d$ linearly independent vectors $v_{1}, \ldots, v_{d}$ from $a_{1}, \ldots, a_{t}$, and after that choose $r-d$ vectors $v_{d+1}, \ldots, v_{r}$ from $a_{t+1}, \ldots, a_{m}$ such that $v_{1}, \ldots, v_{r}$ are linearly independent. Let $V$ be the matrix with rows $v_{1}, \ldots, v_{r}$, and determine, with the algorithm of Kannan and Bachem [5], a unimodular matrix $U$ such that $V U$ is in (lower-triangular) Hermite normal form. One easily checks that $U$ has the required properties.

Now $A x \leqslant 0$ is totally dual integral if and only if $A U x \leqslant 0$ is totally dual integral. So we may assume that $A=A U$, and that $F=\mathbb{R}^{d} \times 0^{n-d}$ and $L=\mathbb{R}^{r} \times 0^{n-r}$. Since now the last $n-r$ columns of $A$ are zero, we may assume that $n=r$. It is easy to see that $A x \leqslant 0$ is totally dual integral if and only if $a_{1}, \ldots, a_{t}$ form a Hilbert basis for $F$, and $A^{\prime} x \leqslant 0$ is totally dual integral, where $A^{\prime}$ is the matrix consisting of the last $n-d$ columns of $A$.

Now the fact that $F$ is a linear space implies that $a_{1}, \ldots, a_{t}$ is a Hilbert basis for $F$ if and only if $F$ is the convex cone generated by $a_{1}, \ldots, a_{t}$ (which is a given fact, by definition of $a_{1}, \ldots, a_{t}$ ), and $F \cap \mathbb{Z}^{n}$ is the lattice generated by $a_{1}, \ldots, a_{t}$ (which can be checked again with the algorithm of Kannan and Bachem). Indeed, necessity of these conditions is immediate. To see sufficiency, let $z$ be an integer vector in $F$. Then $z=\nu_{1} a_{1}+\cdots+\nu_{t} a_{t}$ for certain integers $\nu_{1}, \ldots, \nu_{t}$. Moreover, for each $i=$ $1, \ldots, t$, the vector $-a_{i}$ belongs to $F$, and can hence be written as a convex combination of $a_{1}, \ldots, a_{t}$. Therefore, $0=\mu_{1} a_{1}+\cdots+\mu_{t} a_{t}$ for positive rationals $\mu_{1}, \cdots, \mu_{t}$. By choosing $M$ appropriately, $z=\left(\nu_{1}+M \mu_{1}\right) a_{1}+\cdots+\left(\nu_{t}+M \mu_{t}\right) a_{t}$ is a decomposition of $z$ as a nonnegative integer combination of $a_{1}, \ldots, a_{t}$. 
So it suffices to describe a test of total dual integrality for $A^{\prime} x \leqslant 0$. That is, without loss of generality we may assume that the cone $C$ generated by $a_{1}, \ldots, a_{m}$ is full-dimensional and pointed.

II. Here we describe an algorithm to test whether $a_{1}, \ldots, a_{m}$ is a Hilbert basis for the cone $C$ generated by $a_{1}, \ldots, a_{m}$, where $C$ is pointed and full-dimensional.

We first observe that $a_{1}, \ldots, a_{m}$ is a Hilbert basis for $C$ if and only if the only integer vector in the set

$$
C_{0}=\left\{z \in C \mid z-a_{i} \notin C \text { for } i=1, \ldots, m\right\}
$$

is the origin.

To prove necessity, let $z$ be an integer vector in $C_{0}$. Then $z=\lambda_{1} \alpha_{1}+\cdots+\lambda_{m} a_{m}$ for nonnegative integers $\lambda_{1}, \ldots, \lambda_{m}$. As $z-a_{i} \notin C$ we know that $\lambda_{1}=\cdots=\lambda_{m}=0$, i.e., that $z=0$.

To prove sufficiency, let $z$ be an integer vector in $C$. Let $\lambda_{1}$ be the highest rational number such that $z-\lambda_{1} a_{1}$ belongs to $C$. Next, let $\lambda_{2}$ be the highest rational number such that $z-\left\lfloor\lambda_{1}\right\rfloor a_{1}-\lambda_{2} a_{2}$ belongs to $C$. If $\lambda_{1}, \ldots, \lambda_{j}$ have been found, let $\lambda_{j+1}$ be the highest rational number such that $z-\left\lfloor\lambda_{1}\right\rfloor a_{1}-\left\lfloor\lambda_{2}\right\rfloor a_{2}-\cdots-\left\lfloor\lambda_{j}\right\rfloor a_{j}-\lambda_{j+1} a_{j+1}$ belongs to $C$. When $\lambda_{1}, \ldots, \lambda_{m}$ have been found, the vector $z-\left\lfloor\lambda_{1}\right\rfloor a_{1}-\cdots-\left\lfloor\lambda_{m}\right\rfloor a_{m}$ is an integer vector in $C_{0}$, and hence is the origin. This expresses $z$ as a nonnegative integer combination of $a_{1}, \ldots, a_{m}$.

So it suffices to check whether the only integer vector in $C_{0}$ is the origin. To this end let $b_{1}, \ldots, b_{1}$ be vectors such that

$$
C=\left\{z \mid b_{j} z \geqslant 0 \text { for } j=1, \ldots, t\right\}
$$

Since the rank $r=n$ of $a_{1}, \ldots, a_{m}$ is fixed, such $b_{1}, \ldots, b_{t}$ can be found in polynomial time (as each facet of $C$ is determined by $r$ linearly independent vectors from $\left.a_{1}, \ldots, a_{m}\right)$.

Now it follows trivially from (3) and (4) that

$$
C_{0}=\left\{z \in C \mid \text { for all } i=1, \ldots, m \text { there exists } j=1, \ldots, t \text { with } b_{j} z<b_{j} a_{i}\right\} .
$$

So if $\Phi$ denotes the collection of all functions $\phi:\{1, \ldots, m\} \rightarrow\{1, \ldots, t\}$, then

$$
C_{0}=\bigcup_{\phi \in \Phi}\left\{z \mid b_{j} z \geqslant 0 \text { for } j=1, \ldots, t \text {, and } b_{\phi(i)} z<b_{\phi(i)} a_{i} \text { for } i=1, \ldots, m\right\} \text {. }
$$

This expresses $C_{0}$ as a union of convex sets, and we have to test whether the only integer vector in each of these convex sets is the origin. Below we shall see that this can be done in polynomial time (for fixed dimension) with Lenstra's algorithm for integer linear programming [7]. (Note that $\Phi$ generally has exponential size, even for fixed rank $r$ of $a_{1}, \ldots, a_{m}$.)

Let $Z$ be the collection of vectors $z$ determined by $n$ linearly independent equations from:

$$
\begin{array}{ll}
b_{j} z=0 & (j=1, \ldots, t), \\
b_{j} z=b_{j} a_{i} & (j=1, \ldots, t ; i=1, \ldots, m) .
\end{array}
$$


Since $n$ is fixed, we can enumerate and store $Z$ in polynomial time. Next let $\Sigma$ be the collection of all subsets $\left\{z_{1}, \ldots, z_{n}\right\}$ of $Z$ such that:

(i) $z_{1} \ldots, z_{n}$ are linearly independent,

(ii) $z_{1}, \ldots, z_{n}$ belong to $C$,

(iii) $\forall i=1, \ldots, m \exists j=1, \ldots, t \forall k=1, \ldots, n: b_{j} z_{k} \leqslant b_{j} a_{i}$.

Again, $\Sigma$ can be enumerated and stored in polynomial time. Define

$$
\sigma\left(z_{1}, \ldots, z_{n}\right)=\text { convex hull }\left\{0, z_{1}, \ldots, z_{n}\right\} \mid \text { convex hull }\left\{z_{1}, \ldots, z_{n}\right\},
$$

for $\left\{z_{1}, \ldots, z_{n}\right\}$ in $\Sigma$. We finally show:

$$
C_{0}=\bigcup_{\left\{z_{1}, \ldots, z_{n}\right\} \in \Sigma} \sigma\left(z_{1}, \ldots, z_{n}\right) \text {. }
$$

we are finished as soon as we have proved (10): with Lenstra's algorithm we can test, for each $\left\{z_{1}, \ldots, z_{n}\right\}$ in $\Sigma$, whether $\sigma\left(z_{1}, \ldots, z_{n}\right) \mid\{0\}$ contains integer vectors. Hence we can test whether $C_{0}$ contains integer vectors other than the origin.

To prove (10), first observe that $C_{0}$ is bounded. Indeed, $C_{0} \subseteq\left\{\lambda_{1} a_{1}+\cdots+\right.$ $\lambda_{m} a_{m} \mid 0 \leqslant \lambda_{i}<1$ for $\left.i=1, \ldots, m\right\}$. Now let $w \in C_{0}$. Then by (6) there exists a function $\phi$ in $\Phi$ such that $w$ belongs to the convex set

$$
P=\left\{z \mid b_{j} z \geqslant 0 \text { for } j=1, \ldots, t \text {, and } b_{\phi(i)} z<b_{\phi(i)} a_{i} \text { for } i=1, \ldots, m\right\} .
$$

Since $P$ is bounded and nonempty, it remains bounded if we replace in (11) the sign $<$ by $\leqslant$, thus obtaining the closure $\bar{P}$ of $P$. Since $w \in P$ there exists an $\varepsilon>0$ such that $(1+\varepsilon) w$ belongs to $P$. As $0 \in \bar{P},(1+\varepsilon) w$ is a convex combination of some linearly independent vertices $z_{1}, \ldots, z_{n}$ of $\bar{P}$. Then $\left\{z_{1}, \ldots, z_{n}\right\} \in \Sigma$ and $w \in$ $\sigma\left(z_{1}, \ldots, z_{n}\right)$.

To prove the reverse inclusion for (10), let $\left\{z_{1}, \ldots, z_{n}\right\} \in \Sigma$ and $w \in \sigma\left(z_{1}, \ldots, z_{n}\right)$. There exists $\varepsilon>0$ such that $(1+\varepsilon) w \in \sigma\left(z_{1}, \ldots, z_{n}\right)$. By (8) (ii) $w$ belongs to $C$. Moreover, by (8) (iii), there exists a function $\phi$ in $\Phi$ such that $b_{\phi(i)} z_{k} \leqslant b_{\phi(i)} a_{i}$ for $i=1, \ldots, m$ and $k=1, \ldots, n$. Since $b_{j} z_{k} \geqslant 0$ for all $j=1, \ldots, t$ and $k=1, \ldots, n$, and since $z_{1}, \ldots, z_{n}$ are linearly independent, we know that $b_{\phi(i)} a_{i}>0$ for all $i=1, \ldots, m$. Therefore, $(1+\varepsilon) b_{\phi(i)} w \leqslant b_{\phi(i)} a_{i}$ for $i=1, \ldots, m$, implies that $b_{\phi(i)} w<b_{\phi(i)} a_{i}$ for $i=1, \ldots, m$, and hence that $w$ belongs to $C_{0}$.

The problem is still open whether testing total dual integrality is co-NP complete or polynomially solvable (or both).

\section{References}

[1] R. Chandrasekharan, "Polynomial algorithms for totally dual integral systems and extensions", Annals of Discrete Mathematics 11 (1981) 39-51. 
[2] R. Chandrasekharan and S. Shirali, "Total weak unimodularity: Testing and applications", Report University of Texas at Dallas, 1981.

[3] J. Edmonds and R. Giles, "A min-max relation for submodular functions on graphs", Annals of Discrete Mathematics 1 (1977) 185-204.

[4] F.R. Giles and W.R. Pulleyblank, "Total dual integrality and integer polyhedra", Linear Algebra and Its Applications 25 (1979) 191-196.

[5] R. Kannan and A. Bachem, "Polynomial algorithms for computing the Smith and Hermite normal forms of an integer matrix", SIAM Journal on Computing 8 (1979) 499-507.

[6] L.G. Khachiyan, "A polynomial algorithm in linear programming", Doklady Akademii Nauk SSSR 244 (1979) 1093-1096 (English translation: Soviet Mathematics Doklady 20 (1979) 191-194).

[7] H.W. Lenstra, Jr., "Integer programming with a fixed number of variables", Mathematics of Operations Research 8 (1983) 538-548.

[8] A. Schrijver, "On total dual integrality", Linear Algebra and Its Applications 38 (1981) 27-32. 